\title{
Estudio del Secado de Anacardo (Anacardium occidentale L.) mediante Secador Solar de Radiación Directa
}

Antônio V. Machado*, Edson L. Oliveira, Everaldo S. Santos y Jackson A. Oliveira Universidade Federal do Rio Grande do Norte-UFRN, Programa de Pós-Graduação em Engenharia Química - PPGEQ, Núcleo Tecnológico, Campus Universitário s/n, 59072-970 Natal, RN-Brasil (e-mail: machadoav@eq.ufrn.br).

*autor a quien debe ser dirigida la correspondencia

\section{Resumen}

Se presentan ensayos de secado con radiación solar directa y su modelado matemático, para evaluar la cinética de secado del anacardo (Anacardium occidentale L.) y comparar su eficiencia en relación al secado solar natural en tablero. Los experimentos fueron realizados con rodajas de fruta de $1 \mathrm{~cm}$. y $2 \mathrm{~cm}$. de espesor, obteniendo su contenido acuoso versus tiempo. Con el empleo del secador solar de radiación directa, se redujo el tiempo de secado en relación al secado natural en tablero, agregando valor a los productos. Se obtuvo valores de difusividad de agua en rodajas de frutos del mismo orden de otros datos de la literatura. Los productos secados resultaron tener buenas propiedades organolépticas, tales como sabor, aroma, color y textura crocante.

Palabras clave: secador solar, anacardo, Anacardium occidentale L., radiación directa

\section{Cashew-nut (Anacardium occidentale L.) Drying Study Using a Solar Dryer with Direct Radiation}

\begin{abstract}
In this work solar drying tests with direct radiation and its mathematical modeling to evaluate the kinetics of cashew-nut (Anacardium occidentale L.) drying comparing their efficiency concerned to natural solar drying board are presented. The experiments were performed using fruit slices with 1 $\mathrm{cm}$ and $2 \mathrm{~cm}$ of thickness, obtaining its water content versus time. Using a direct solar radiation dryer, the drying time was lower than that of natural drying board, therefore adding value to products. Diffusivity values obtained from water sliced fruits were similar to other data available in the literature. The dried products had good organoleptic properties, aroma, color and crunchy texture.
\end{abstract}

Keywords: solar drying, cashew-nut, Anacardium occidentale L., direct radiation 


\section{INTRODUCCIÓN}

El anacardo (Anacardium occidentale L.) es un árbol frutal originario de Brasil y que se puede encontrar en varios países de clima tropical. Los mayores productores mundiales de anacardo según datos del IBGE (2008), son: Vietnam, China y Brasil. Según Andrigueto y Kososki (2006), la región Nordeste de Brasil tiene un área plantada superior a las 736 mil hectáreas, siendo los estados de Rio Grande do Norte, Ceará, Piauí, y Bahía, los principales productores. Los estados de Rio Grande do Norte y Ceará destacan entre los mayores productores de anacardo del Nordeste presentando una producción de 265.888 ton de castaña de anacardo en el año de 2007. Su fruto (castaña) y pseudofruto (pedúnculo hipertrofiado) que corresponde a $90 \%$ del peso del anacardo son ampliamente utilizados en la industria alimenticia. Sin embargo, el carácter perecedero del pedúnculo, el cual es bastante consumido "in natura", dificulta considerablemente su comercialización en los mercados más distantes de los centros de producción. Además de frágil, es altamente perecedero, ya que sufre por mecanismos aceleradores de degradación microbiológica contribuyendo así la pérdida de millones de toneladas de producto. La gran cantidad desperdiciada, de aproximadamente 2.393.000 ton en el año de 2007, representa cerca del 94\% de la producción total, lo cual, por otra parte presentaría un elevado potencial de aprovechamiento (Machado et al., 2008).

La pulpa del pedúnculo del anacardo es una materia prima rica en carbohidratos, fibras, vitaminas y sales minerales (Souza et al., 2005). El pedúnculo es de alto valor nutritivo y por unidad es uno de los vegetales más ricos del mundo en vitamina $\mathrm{C}$, y quizás sea el fruto más barato entre todos los cultivados en Brasil. Analizando el zumo de anacardo se observó que en un mismo volumen de este zumo contiene cerca del $164,2 \mathrm{mg} / 100 \mathrm{~g}$ de vitamina C, 4 a 5 veces más vitamina $\mathrm{C}$ que el zumo de naranja que contiene cerca del $32,8 \mathrm{mg} / 100 \mathrm{~g}$ de vitamina $\mathrm{C}$, Ifruta con alto contenido en vitamina $\mathrm{C}$ (Souza, 2007). Se debe destacar que Brasil es el único país del mundo que tiene el hábito de consumo del pedúnculo in natura, en sus diferentes formas como fruto de tabla, con lo que al menos en Brasil, sería un negocio prometedor y una alternativa a su aprovechamiento contribuiría a la reducción del elevado índice de desechos (Alsina, 2004).

La preservación de alimentos por secado es uno de los métodos más antiguos del mundo y es utilizado por el hombre hasta hoy. Algunos alimentos como granos y semillas ya están bastantes secos cuando son cosechados, permanecen en buenas condiciones por un buen periodo de tiempo (Vega, 2005). En la conservación de alimentos, la deshidratación o secado es considerado como uno de los procedimientos importantes en la reducción de la actividad del agua (Aw). Sin embargo la mayoría de los alimentos contiene una humedad elevada, lo que permite la acción de microorganismos y él secado se hace necesario en el caso estudiado en el presente trabajo. En los alimentos secos los microorganismos prácticamente no se desarrollan debido a la baja actividad del agua. Asimismo se inhibe la mayoría de las reacciones químicas y enzimáticas que provocan alteraciones en los alimentos (Gaspareto, 2004), favoreciendo el transporte y la manipulación del producto, además de prolongar la vida de almacenamiento (Emepa, 2007).

La energía solar se presenta como alternativa de gran interés por sus cualidades y características de ser limpia, de gran potencial, altamente disponible en todo Brasil y sobre todo en la región Nordeste, donde se tiene sol casi todo año. El desarrollo de tecnología para el aprovechamiento de la energía solar, utilizando equipos que puedan transformar energía solar en calor es extremadamente importante en el momento actual. Se justifica también el uso de la energía solar por la escasez y gran poder contaminante de las fuentes de energía fósiles normalmente utilizadas (Souza, 2007).

Dentro de los diferentes sistemas de secado de productos alimentarios, el secado solar tradicional es, todavía hoy, el procedimiento más utilizado en todo el mundo, sobre todo cuando se trata del secado de granos, frutos y semillas utilizando sistemas del tipo terreno de suelo apisonado y tablero. En un sistema de secado simple usando energía solar, el secador solar de radiación directa (utilizado en este trabajo) es un sistema de bajo costo, eficiente y bastante utilizado en el secado de alimentos. Por otro lado, este sistema tiene como inconveniente la dificultad del control de las condiciones operativas como: control de temperatura, velocidad y humedad relativa del aire, ya que tales parámetros dependen de las condiciones climatológicas. El secador de radiación directa está 
compuesto por un cajón rectangular de metal aislado con lana de vidrio, cubierto con un vidrio transparente donde incide la energía solar y en su parte inferior una placa de metal pintada de negro para absorción de la energía incidente. El secado del pedúnculo de anacardo en secadores solares puede ser una alternativa para los pequeños productores ya que usando esta tecnología pueden agregar valor a sus productos, minimizar las pérdidas de producción y aumentar la renta familiar. Así, la utilización de sistemas solar en el secado de alimentos puede contribuir en la fijación de población en el campo, evitando así el éxodo rural. El anacardo presenta un enorme potencial para obtención de productos deshidratados a considerando su alto desperdicio, y no se encuentran habitualmente trabajos sobre el secado anacardo utilizando secadores.

El secado de alimentos tanto de origen animal como vegetal requiere un estudio minucioso de las condiciones de operación, teniendo en cuenta las características deseadas en el producto final (Honorato, 2005). La cinética del secado debe ser bien definida con relación a los efectos de las propiedades del material y del medio de secado, en particular por medio de las propiedades de transporte, como conductividad y difusividad térmica, difusividad másica, coeficientes de transferencia de calor y masa (Sokhansanj, 1994). Sin embargo, los modelos usados para simular la cinética del secado, también pueden ser utilizados para diseñar unidades nuevas, así como para controlar y optimizar unidades ya existentes (Karathanos y Belessiotis, 1999). Teniendo en cuenta lo expuesto se puede afirmar que las frutas deshidratadas poseen un mercado mundial con gran potencial de crecimiento y que hasta la fecha es poco explotado económicamente en Brasil.

A partir de la segunda Ley de Fick, es posible obtener modelos semi-empíricos para determinar el coeficiente de difusión efectivo $\left(D_{\mathrm{ef}}\right)$ en geometría de placa plana infinita y con diversas condiciones de contorno y de operación (Crank, 1975).

$$
\frac{d U}{d t}=D e f \frac{\partial^{2} U}{\partial u^{2}}
$$

Una de las dificultades para analizar datos de difusión en el secado, es que la condición inicial para el periodo con control difusional corresponde a una distribución no uniforme de humedad en el sólido, especialmente cuando el periodo de velocidad constante precede al periodo de velocidad decreciente.

Durante el secado convectivo, con velocidad del aire suficientemente elevada, la resistencia externa es generalmente muy pequeña y la velocidad de secado es controlada por la difusión en el sólido. En estas condiciones, durante el período de velocidad decreciente, la humedad en la superficie está en equilibrio con el medio de secado y no hay agua libre.

Considerando que la distribución inicial de humedad es uniforme ( $U=U$ o en $t=0)$ y que la superficie está en equilibrio con el medio de secado, (U=Ue en $u= \pm L / 2)$ la ecuación (1) se integra:

$$
R U=\frac{U(t)-U e}{U o-U e}=\frac{8}{\pi^{2}} \sum_{i=0}^{\infty} \frac{1}{(2 i+1)} \exp \left[-\frac{(2 i+1)^{2} \cdot \pi^{2} \cdot \text { Def } . t}{4 L^{2}}-\right]
$$

La ecuación (2), que describe la evolución temporal de la humedad media de un cuerpo sometido a secado, se obtiene por integración en el espacio de la solución de la segunda ley de Fick con las siguientes consideraciones: geometría de placa plana infinita, transferencia unidireccional de masa, la resistencia externa convectiva es mucho menor que la resistencia difusional interna, lo que permite suponer un contenido de agua en la interfase constante e igual al valor de equilibrio con el aire circundante, el coeficiente efectivo de difusión constante y distribución inicial de humedad uniforme.

El objetivo de este trabajo es evaluar la cinética del proceso de secado del anacardo, en secador solar de radiación directa, utilizando rodajas de fruta de $1 \mathrm{~cm}$ y $2 \mathrm{~cm}$ de espesor, verificando su eficiencia en relación al método de secado solar natural en tablero. 


\section{MATERIALES Y MÉTODOS}

Para realización de este trabajo habían sido utilizados pseudofrutos del anacardo previamente seleccionados de acuerdo con su grado de maduración, coloración de la piel y ausencia de daños físicos. Tras lavado y saneado de los pseudofrutos fueron retiradas las castañas y cortados en rodajas de 1 y $2 \mathrm{~cm}$. de espesor. El contenido de humedad del material a ser secado se determinó por el método tradicional de pesaje en estufa, a $70^{\circ} \mathrm{C}$, hasta llegar a un peso constante.

Los equipos utilizados para el secado de las rodajas de anacardo fueron un secador solar de radiación directa y un secador del tipo tablero (usado como patrón) desarrollado en el PPGEQ de la Universidade Federal do Rio Grande do Norte-UFRN. El secador solar de radiación directa está constituido de un cajón rectangular de metal con apertura superior e inferior, protegida por una pantalla que permite el paso de aire mediante convección natural. La materia prima se dispone en una bandeja (la parte inferior de la bandeja en tela metálica de malla fina y todo el conjunto confeccionado en acero inoxidable) que es puesta en el centro del secador. El secador de tablero está constituido de una bandeja con parte inferior en tela metálica de malla fina (todo el conjunto de acero inoxidable), puesto sobre un caballete de metal expuesto a la radiación solar directa. En intervalos regulares de tiempo, se retiraban las bandejas de los secadores, eran pesadas rápidamente y recolocadas en los mismos, este procedimiento fue repetido hasta que el material alcanzó el equilibrio higroscópico de humedad, es decir, no se perdía más humedad en las condiciones de operación descrita.

El coeficiente de difusión efectiva fue determinado utilizándose la solución analítica de la ecuación de la segunda ley de Fick (Ec.2), para placa plana, asumiendo que la humedad migra solamente por difusión y la contracción es despreciable (Crank, 1975).

$R U=\frac{U(t)-U e}{U o-U e}=\frac{8}{\pi^{2}} \sum_{i=0}^{\infty}-\frac{1}{(2 i+1)} \exp \left[-\frac{(2 i+1)^{2} \cdot \pi^{2} \cdot \text { Def } . t}{4 L^{2}}-\right]$

Donde:

$R U$ = es la razón de humedad (adimensional);

$U(t)=$ es la variación de la humedad (\% b.s.) con el tiempo;

Ue = es la humedad de equilibrio (\% b.s.);

Uo = es la humedad inicial (\%b.s.);

Def $=$ es el coeficiente de difusibid efectiva $\left(\mathrm{m}^{2} \mathrm{~s}^{-1}\right)$ y $\mathrm{L}$ es el espesor de la rodajas $(\mathrm{m})$.

Las propiedades sensoriales fueron evaluadas por probadores entrenados analizando los atributos de sabor, aroma, textura y apariencia del producto seco.

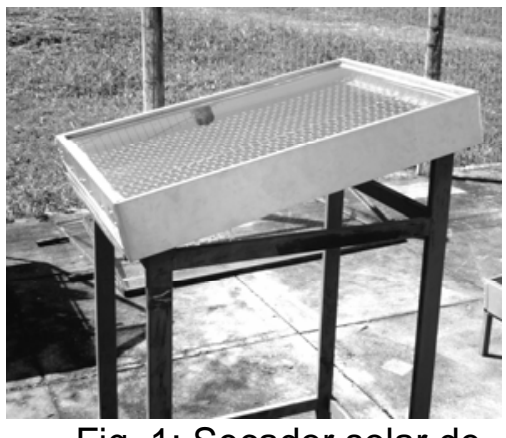

Fig. 1: Secador solar de radiación directa.

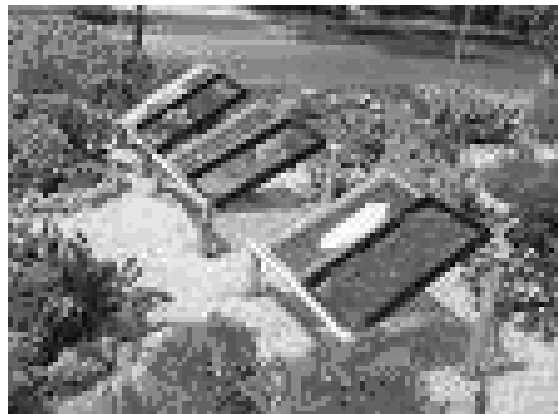

Fig. 2: Secador solar del tipo tablero.

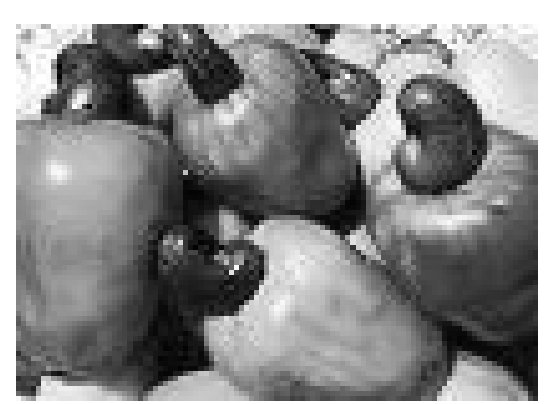

Fig. 3: Pseudofrutos del anacardo.

El experimento fue realizado con un diseño totalmente aleatorio, con cinco repeticiones, y consistió en tratamientos de secado con secador solar de radiación directa (SSRD) y secador solar del tipo tablero (SST), variando el espesor de la rodajas en $1 \mathrm{~cm}$ y $2 \mathrm{~cm}$, terminando el esquema factorial 
(2x2). Los datos fueron analizados por el programa de ordenador "Sistema para Análisis de Variancia SISVAR", utilizando el índice de $5 \%$ de probabilidad en el test de Tukey para comparación de las medianas. Las curvas de secado fueron sometidas al análisis de variancia estudiándose los valores en función del tiempo de secado a través del análisis de regresión. La Fig. 1,2 y 3, se representa el dispositivo experimental secador solar de radiación directa, secador solar del tipo tablero y pseudofrutos del anacardo.

\section{RESULTADOS Y DISCUSIÓN}

Las rodajas de anacardo obtenidas tras el proceso de secado solar presentaron buenas características sensoriales, con aroma y sabor agradable, con buen aspecto visual general de las rodajas secas y color amarillo claro, sin vestigios de oscurecimiento y manteniendo también las características rojizas de la cáscara.

Las rodajas de anacardo presentaron textura crujiente y no presentaron consistencia elástica. Esto está relacionado con la baja higroscopicidad del material seco en las condiciones del presente trabajo. Resultados semejantes a los obtenidos por (Aragão et al., 2007) en el estudio realizado sobre secado de anacardo en secadores convectivos utilizando calentadores eléctricos.

Los resultados de la Tabla 1, muestran que el espesor de las rodajas de anacardo es un factor limitativo en el tiempo de secado. El menor tiempo de secado fue obtenido por el tratamiento secador solar de radiación directa (SSRD), con rodajas de espesor de $1 \mathrm{~cm}$ llegando al final del proceso con un tiempo de 23 horas, seguido por el mismo tratamiento con espesor de la rodajas de $2 \mathrm{~cm}$ y 33,5 horas de secado. En el tratamiento por secador solar del tipo tablero (SST), con rodajas de $1 \mathrm{~cm}$ de espesor se finalizó en un tiempo de 40 horas. El mismo tratamiento con rodajas de $2 \mathrm{~cm}$ de espesor fue el que tuvo el mayor tiempo de secado, 51 horas. El análisis estadístico probó una diferencia significativa entre todos los tratamientos.

Tabla 1: Valores medios del tiempo final (en horas) de secado solar de rodajas de anacardo, en función de los diferentes tratamientos. [Medianas seguidas por letras distintas en la misma columna, difieren entre sí por el test de Tukey, $(p=0,05)]$

\begin{tabular}{ccc}
\hline Tratamientos & $\begin{array}{c}\text { Espesor de la rodajas } \\
(\mathrm{cm})\end{array}$ & $\begin{array}{c}\text { Tiempo final de secado } \\
\text { (horas) }\end{array}$ \\
\hline SSRD & 1 & $23,0 \mathrm{~d}$ \\
SSRD & 2 & $33,5 \mathrm{c}$ \\
SST & 1 & $40,0 \mathrm{~b}$ \\
SST & 2 & $51,0 \mathrm{a}$ \\
\hline
\end{tabular}

El comportamiento cinético del proceso de secado está representado en la Fig. 4. Las curvas de secado solar presentaron el mismo comportamiento cinético tanto para el tratamiento secador solar de radiación directa como para el secador solar del tipo tablero. Ambos presentaron un decrecimiento acentuado de la humedad en el inicio del secado, en seguida la humedad es reducida moderadamente y estabilizando al final del proceso hasta llegar al equilibrio. Mediante la comparación, de las curvas obtenidas en el proceso de secado, se puede observar que el tiempo de procesamiento disminuye significativamente con la reducción del espesor de las rodajas de anacardo y el tipo de secador utilizado. Los resultados experimentales sugieren la aplicabilidad del modelo Fickiano simple, (Ec.2). Se observan en la Tabla 2, los valores de $\mathrm{R}^{2}$ de las curvas experimentales y se observa también, que los coeficientes de difusividad efectiva variaron entre $1,97 \times 10^{-8}$ y $3,94 \times 10^{-8}$. Estos resultados se mostraron próximos a los reportados por (Oliveira et al., 2005) en estudio sobre la cinética de secado del anacardo en secador convectivo con difusividad efectiva variando entre $1,71 \times 10^{-07}$ y $1,97 \times 10^{-07}$. Mediante la comparación de las curvas de secado obtenidas se verifica que la tasa de secado aumenta con la disminución del espesor de las rodajas de anacardo, conforme los coeficientes de difusión efectiva determinados. Tabla 2. Valores medianos de $A, K, R^{2} y$ Coeficiente de difusividad para secado solar de rodajas de anacardo. 


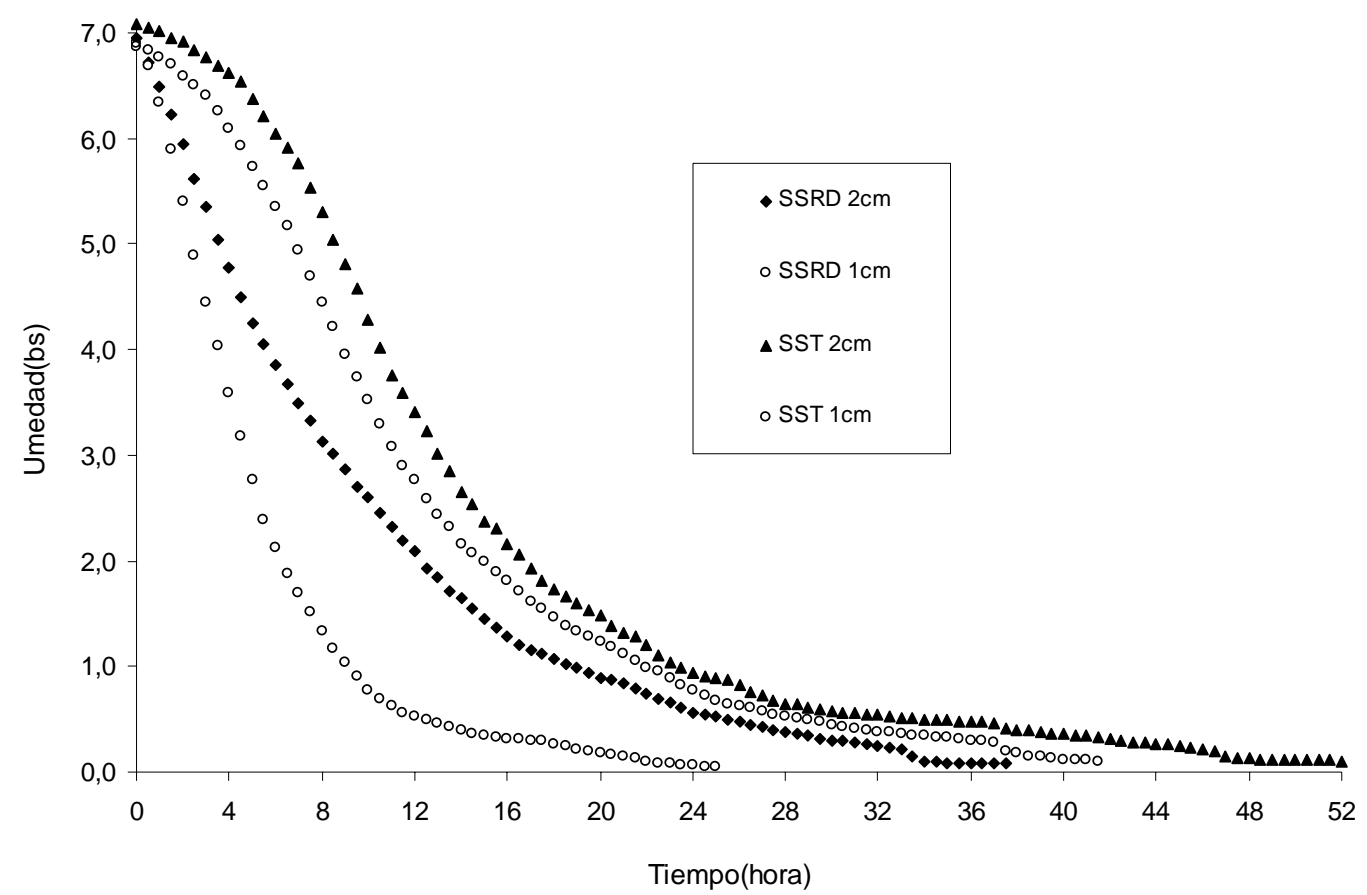

Fig. 4: Curva de secado solar de anacardo en rodajas en secador de radiación directa y secador natural de tablero.

Tabla 2. Valores medianos de $A, K, R^{2}$ y Coeficiente de difusividad para secado solar de rodajas de anacardo.

\begin{tabular}{cccccc}
\hline Tratamientos & $\begin{array}{c}\text { Espesor de las rodajas } \\
(\mathrm{cm})\end{array}$ & $\mathrm{A}$ & $\mathrm{K}\left(\mathrm{min}^{-1}\right)$ & $\mathrm{R}^{2}$ & Def. $\left(\mathrm{m}^{2} / \mathrm{s}\right)$ \\
\hline SSRD & 1 & 0,671 & 0,2329 & 0,993 & $3,94 \mathrm{E}-08$ \\
SSRD & 2 & 0,891 & 0,0432 & 0,997 & $2,92 \mathrm{E}-08$ \\
SST & 1 & 0,652 & 0,1226 & 0,991 & $2,07 \mathrm{E}-08$ \\
SST & 2 & 0,515 & 0,0291 & 0,989 & $1,97 \mathrm{E}-08$ \\
\hline
\end{tabular}

\section{CONCLUSIONES}

La secado de anacardo en sistemas solar demostró obtener productos con buenísimas propiedades sensoriales de sabor, aroma, color y presentando textura crujiente.

El espesor de las rodajas de anacardo es un factor crucial en el tiempo de secado donde se verifica que la velocidad de secado aumenta con la disminución del espesor de las rodajas. Los coeficientes de difusión efectiva encontrados variaron entre $1,97 \times 10^{-8}$ y $3,94 \times 10^{-8}$.

El secador solar de radiación directa se presenta como una alternativa viable y de gran importancia para la conservación del pedúnculo de anacardo, reduciendo el tiempo de secado considerablemente en relación al secador solar del tipo tablero.

\section{AGRADECIMIENTOS}

Al CNPQ por el soporte financiero.

\section{REFERENCIAS}

Alsina, O.I.S.; Estudos das propriedades térmicas da goiaba (psidium guajava l.) e do caju(anacardium occidentalle I.) Congresso Brasileiro de Engenharia Química, v.15, p. 44-49, Curitiba (2004). 
Andrigueto, J. R. y A.R. Kososki; Desenvolvimento e conquista da produção integrada de frutas no Brasil. In: Manual de Produção integrada de Caju. EMBRAPA, v.3, p. 225, (2006).

Aragão, R.F., O.L.S. Asina y A.M. Guedes; Estudo experimental da secagem de fatias de caju. In: Alimentos Ciencia e Ingeniería: 16(3), 302-307 (2007).

Crank, J.; The mathematics of diffusion. $2^{\mathrm{a}}$ edition. Claredon Pres Oxford, New York (1975).

Emepa. Caju < http://www.emepa.org.br/sigatoka.php > Data de Edição: 25/06/07. Acesso em 14 de outubro de 2008.

Gaspareto, O.C.P.; Banana e mamão estudo cinético modelagem e simulação Jaca tratada osmóticamente e caroços secos para produção de farinha. Natal, UFRN: Tese Doutorado em Engenharia Química, p.248 (2004).

Honorato, G.C. y E.L. Oliveira;. Estudio del Proceso Cinético del Secado de Cefalotórax de Camarón. Información Tecnológica, 16(4), 3-10 (2005). Disponível em: <http://www.scielo.cl/scielo.php? script=sci_arttext\&pid=S0718-642005000400002\&lng=es\&nrm=iso>. Acesso em: 09 Feb. de 2009.

IBGE, Instituto Brasileiro de Geografia e Estatística. Disponivel em http://www.ibge.gov.br/news.php? recid=355. Data de Edição: 21/01/08. Acesso em 25 de agosto de 2008.

Karathanos, V.T. y V.G. Belessiotis; Application of a thin-layer equation to Drying Data of Fresh and Semi-dried fruits. Journal Agriculture Eng. Research: 74, 355-361 (1999).

Machado, A.V., E.L. Oliveira, E.S. Santos y J.A. Oliveira; Influência da espessura com o tempo de secagem em sistemas solar de radiação direta e indireta. Congresso Brasileiro de Ciência e Tecnologia de Alimentos (CBCTA), Belo Horizonte - MG, v.3, p. 44-51 (2008).

Oliveira, F.M.N.; A.S. Silva y F.A.C. Alemida; Influência do branqueamento no processo da cinética de secagem do caju. In: I Simpósio Brasileiro de Pós-Colheita de Frutos Tropicais (SBPCFT), v.4, p. 23-29 João Pessoa-PB, (2005).

Sokhansaj, S.; Grain drying simulation with respect to energy conservation and grain quality. 1994. In: Mujumdar, A. S. Advances in drying. Hemisphere, Washington, vol. 3, chapter4, pp. 113-159, (1994).

Souza, M.C.M., T.H.S. Rodrigues, M.V.P. Rocha y L.R.B. Gonçalves; Cinética de secagem do caju. In: I Simpósio Brasileiro de Pós-Colheita de Frutos Tropicais (SBPCFT), v.4, p. 52-57 João PessoaPB (2005).

Souza, I.G.M.; Obtenção de tomates secos utilizando um sistema de secagem solar construído com materiais alternativos. $8^{\circ}$ Congresso Iberoamericano de Engenharia Mecânica; v.6, p. 135-139, Cusco (2007).

Vega, A., A. Andrés y P. Fito; Modelado de la Cinética de Secado del Pimiento Rojo (Capsicum annuum L. CV Lamuyo). Información Tecnológica, ISSN 0718-0764 (en línea), 16(6), 3-11 (2005).<http://www.scielo.cl/scielo.php?script=sci_arttext\&pid=S071807642005000600002\&lng=es\&n rm=iso>. Acceso: 7 de Enero de 2008. 